\title{
L'enfermement Identitaire Dans Le Cinema Marocain Moderne
}

\author{
Dr. Adil Bouyahya
}

Centre de formation pour les métiers de l'éducation, Fes, Morocco

doi: 10.19044/esj.2016.v12n35p422 URL:http://dx.doi.org/10.19044/esj.2016.v12n35p422

\begin{abstract}
This article analyses the Moroccan cinema during the period (19902000) since it focuses on a central issue of its discursive and esthetic unity. Thus, the article addresses the question of confinement. In other words, in this paper we seek to reflect on the unifying desire and the confined collective identity. In fact, this cinematography deals mainly with a quasiarchaic Moroccan society which is confined in the rigidity of an ArabIslamic identity. Besides, we adopt am esthetic scenographic approach to delineate the Moroccan society's imprisonment within the borders of immutable values. In this paper, the main protagonists in the movies under scrutiny do not enjoy their status as modern heroes; on the contrary, they only access this role when they are deemed as desire objects in quest for a space of freedom and liberty.
\end{abstract}

Keywords: Modern Moroccan cinema, confinement, ancestral values, identity quest

\section{Resume}

En analysant le cinéma marocain de la période 90-2000, on centre notre propos sur ce que nous considérons comme étant son unité discursive et esthétique, à savoir la question de l'enfermement. Nous avons cherché à réfléchir sur la vocation unificatrice et « clôturante » de l'identité collective. La filmographie traite immanquablement d'une société marocaine, quasi archaïque, cloîtrée dans la rigidité du code identitaire arabo-islamique, fermée dans les limites de valeurs immuables. Les personnages principaux de ces films n'accèdent à leur statut de héros modernes que dans la mesure où ils sont des êtres de désir.

Mots Cles: Cinéma marocain moderne, enfermement, valeurs ancestrales, quête identitaire. 
Le présent article propose une recherche qui vise des réponses à des questions. Mais, la recherche entreprise s'effectuera plutôt du côté d'un jeune cinéma qui a réussi néanmoins à faire parler de lui hors de ses frontières : le cinéma marocain.

En analysant le cinéma marocain de la période 90 -2000, à travers une sélection des films les plus représentatifs de la deuxième génération de cinéastes, en l'occurrence Femmes et Femmes et Jawhara, fille de prison de Saâd Chraïbi, Ruses de femmes de Farid BENLYAZID et l'Adieu Forain de Daoued Aouled Syad, en centrant notre propos sur ce que nous considérons comme étant son unité discursive et esthétique, à savoir la question de l'enfermement, nous avons cherché à réfléchir sur la vocation unificatrice et « clôturante » de l'identité collective, c'est-à-dire socioculturelle, et son opposition radicale à toute forme de subjectivité et de singularité individuelle. Or, si les films, tel que nous le montrerons, traitent immanquablement d'une société marocaine, quasi archaïque, cloîtrée dans la rigidité du code identitaire arabo-islamique, fermée dans les limites des valeurs immuables, les personnages principaux des films, n'accèdent à leur statut de héros modernes que dans la mesure où ils sont des êtres de désir. Leur quête de subjectivité, de singularité se déploie dans cette disjonction douloureuse qu'ils vivent, hommes ou femmes, entre un ici où se déploie leur quotidien et un ailleurs vers lequel aspire leur être. Et c'est bel et bien ce désir d'arpenter un ailleurs qui rend l'enfermement dans l'ici aussi insoutenable que récurrent.

Tous les personnages des films expriment, chacun à sa manière, sa quête de différence, son désir de rompre la subordination de la force de vie au principe de l'identité ; rompre l'enfermement, c'est oser sa différence ! Or, s'imposer est déjà un acte violent comme disait Hegel, car un tel acte ne vise rien moins que la souveraineté. Et, à en croire Georges BATAILLE, la souveraineté est l'antre de la raison ; c'est pourquoi il affirme que la souveraineté doit s'expier. Aussi, notre article tente -t-il d'accompagner ce parcours où le principal n'est pas d'aspirer seulement à la souveraineté, mais de la mériter et de l'égaler. Ces héros modernes sont animés par le désir de renoncer à une intégration tributaire de leur assujettissement aux valeurs sociales et culturelles. Leur quête pour joindre l'ouvert de l'ailleurs est mue par un désir de réconciliation avec l'organique qui nous vient de la nature, une réconciliation avec l'instinct qui déloge définitivement le sens et ses injonctions symboliques. Et c’est parce que le désir est si irrévocablement paradoxal, qu'il ne se réalise que dans l'excès et le débordement ; cette lecture intertextuelle des films ne saurait se départir de la tension corrélative à la saisie de cette expression, de cette sensation. Cette recherche, traversée de bout en bout, par notre propre cinéma national, est elle aussi un parcours, une quête personnelle qui, dépassant la simple intelligence de ce 
que l'œuvre voudrait exprimer, s'attache à pénétrer l'œuvre cinématographique marocaine et à lui accorder une valeur pour la réinventer.

De toutes les approches consacrées au cinéma, l'esthétique est celle qui convient le plus à notre objet de réflexion. Evidemment, l'esthétique n'a pas de méthode propre puisqu'il n'existe ni règles, ni méthodes d'analyse clairement établies. Ce que nous entendons par approche esthétique est plutôt un état d'esprit, une attitude d'ouverture face à la force de l'expression. Comme le rappelle Pierre SORLIN, fondée sur une démarche fortement subjective, l'esthétique « se définit à travers les conventions d'une société mais seuls ceux qui la cherchent finissent par la construire ${ }^{20}$. Notre rapport aux films marocains de la deuxième génération, sujet de cet article, est d'abord esthétique parce que l'Intuition, la jouissance, l'émotion, qui sont les premières données de la participation esthétique et la grande manœuvre de subjectivité qu'elle autorise, conviennent parfaitement à notre tempérament. Mais que le lecteur soit rassuré , cette subjectivité et cette participation esthétique ne sont pas les seuls vecteurs de notre réflexion. Même si la tentation de récuser la rigueur au nom de la créativité est grande, nous sommes également conscient qu'une œuvre, même totalement imprévisible, ne se place pas complètement en dehors des règles : la création se fonde sur un matériel convenu, elle a besoin de données de l'expérience : le temps, l'espace, les relations de contiguïté et d'inférence, qu'elle transcode mais qu'elle n'ignore pas. A ce titre, notre approche relève également de la sémiologie, de la narratologie, ou d'autres types d'analyses systématiques. Notre étude esthétique du film marocain serait alors ce lieu paradoxal de l'intuition et de l'interrogation : saisir un film qui nous plaît est un défi que nous voulons relever en acceptant tout ce qui, en lui, englobe et déborde les règles.

En effet, la gageure n'est pas uniquement de monter que le cinéma marocain des années 90-2000 constitue bel et bien une unité narrative et discussive qui se cristallise autour de la problématique de l'enfermement, mais d'expliquer que c'est d'abord, grâce au traitement de l'espace et de l'investissement de la matière filmique, qu'un tel discours se déploie. Et soulignons que la saisie de la dimension structurelle de cette problématique ne nie en rien les singularités spécifiques à chaque film. Quiconque s'intéresse à la production cinématographique des années 90-2000, constatera que les films marocains de cette période traitent immanquablement de l'enfermement. Qu'il s'agisse de l'ignorance qui limite les esprits, de la persistance de traditions figées, des us et coutumes qui coupent les sujets de toute ouverture, ou encore du statut inférieur de la femme, franchement cloîtrée et exclue, les films disent, et quelle que soit l'histoire racontée , la

\footnotetext{
20 - Pierre Sorlin de l'Audiovisuel, Ed. Nathan Université, 1992, p. 13.
} 
douloureuse disjonction que vivent les sujets, entre un ici dysphorique et fermé et un ailleurs désiré et inaccessible.

L'enfermement semble même être une cause immanente à ce cinéma; il dépasserait même le contenu thématique pour concerner la réalité même de la pratique cinématographique de la deuxième génération de cinéastes marocains. En effet, alors que durant la décennie 90 le cinéma de la deuxième génération opérait une connexion inédite avec son public du Maroc et d'ailleurs, il s'est acheminé vers une sorte d'impasse. Dès le début des années 90, les cinéastes marocains de la deuxième génération n'ont pas manqué de souffrir de cette même intransitivité dont a pâti la pratique de leurs prédécesseurs et ce, par une perte progressive du public autochtone et le rétrécissement du rayonnement à l'extérieur.

C'est dire qu'il est évident de constater que l'enfermement est une structure, et que la première partie de ce travail d'analyse consiste à relever les invariants qui procèdent de ce système afin de les cataloguer pour établir une grille de lecture. Néanmoins, l'enjeu nous semble résider ailleurs. En effet, si quelques textes, notamment journalistiques, soulignent la récurrence - jusqu'à la redondance - de cette thématique dans les films marocains de la deuxième génération, ils la confinent dans la seule approche sociologique. Or, il est incontestablement réducteur de maintenir le discours de l'enfermement dans la seule perspective de l'enfermement dans les valeurs de la société patriarcale, c'est-à-dire dans le cadre circonscrit d'une société et d'une culture données, là où, cette notion engage incontestablement des valeurs humaines et ontologiques au-delà de la seule perspective historique. Ceci est d'autant plus regrettable que maintenir l'analyse au niveau du contenu, c'est nier l'essence même de l'expression cinématographique, à savoir la matière filmique.

C'est pourquoi notre postulat est qu'il y a enfermement lorsqu'il y a intransitivité, incommunicabilité ou clôture. Notre premier parti pris a été de situer notre analyse d'abord au niveau de l'expression, dans une approche esthétique qui privilégie de décryptage du système relationnel de composantes filmiques tels : le cadrage, le mouvement, la lumière ou encore le discours verbal, la musique et le bruitage et ce, afin de libérer l'analyse des films marocains de l'emprise de l'unique analyse sociologique et culturelle des contenus des films. Notre préoccupation majeure reste évidemment de maintenir intacte notre hypothèse selon laquelle c'est le traitement de l'espace en relation avec la réalité qui constitue l'unité narrative et discursive de l'ensemble des fims étudiés. Et lorsque nous parlons de récits de l'enfermement, le récit filmique n'est pas entendu comme étant un récit fictionnel mis en images et sons, mais comme des images et des sons agencés en rapport avec le réel, de telle façon qu'ils élaborent un récit. L'espace est alors cette composante essentielle, narrative et signifiante, qui 
engage l'élaboration et le fonctionnement du récit aussi bien sous l'angle de l'énoncé que celui de l'énonciation.

Le lecteur l'aura compris, il n'est pas exclu dans cette réflexion de procéder à une lecture de contenus thématiques en raison même de l'ancrage social et référentiel nettement revendiqué dans les films étudiés, mais le pari consiste à établir de nouveaux entrelacs et de nouvelles copules, aptes à éclairer la notion de l'enfermement par de nouvelles significations et de nouvelles questions.

L'axe principal de ces nouveaux vecteurs étant de saisir les matières expressives par lesquelles l'instance du désir se manifeste comme le lieu paradoxal où les sujets des films sont saisis dans la spirale de l'enfermement et dans leur tentation d'y échapper. L'écriture spatiale de l'enfermement dans les films étudiés a précisément ce mérite d'instaurer une tension créatrice qui déborde les dichotomies discursives conventionnelles du dedans et du dehors, du public et du privé, du sacré et du profane, pour dire qu'il n'y a pas un dehors qui ne soit un dedans et inversement. Et pour saisir cet idéal topique, pour rappeler la citation de Deleuze qui ouvre cette introduction, analyser des films, c'est faire pénétrable à cet en deçà ou au-delà de la représentation, à cette expressivité mouvante et imagée qui suggère si bien la réversibilité des choses, des états et des êtres : la versatilité du désir de se libérer de la possessivité de l'autre et de l'insoutenable solitude de ne pas être possédé .

Quitter la ville traditionnelle ou demeurer dans ces profondeurs, estce vraiment pour le sujets des films, une question topologique, une décision volontaire et discursive, ou bien s'agit-il plutôt pour eux de pousser le désir de l'Ouvert, quand on n'a connu qu'un érotisme de la promiscuité et du fusionnel ?

Désormais, nous pouvons avancer que le désir des sujets des films de se libérer de la société patriarcale, et de sa figure emblématique, le Père, d'échapper à ses valeurs de l'intériorité et de l'affect, à ses lois de l'identification et de l'appartenance sociale, est un désir contrarié, contradictoire et jamais abouti. Se manifestant dans le désordre et le débordement, il bute sur la tension qu'il génère entre la réalisation du Moi et le contentement du Nous. C'est ce côté irréductiblement paradoxal du désir qui peut d'ailleurs expliquer l'attitude équivoque des réalisateurs mêmes des films en question. En effet, il nous semble que Chraïbi, Benlyazid, Ouled Syad, pour ne citer qu'eux, sont également saisis par ce désir paradoxal d'échapper aux impératifs du discours tout en souciant fortement des diktats de l'histoire. En analysant le double rapport du discours de l'enfermement à la question esthétique du réalisme dans le cinéma et à la question sociologique comme rapport au référent, nous souhaitons montrer combien la culture est, paradoxalement, le chemin obligé mais aussi 
l'obstacle à la saisie du réel. Car l'un des enjeux de cette analyse est de porter un regard critique sur le cinéma marocain, un regard qui souligne ses mérites et interroge ses limites.

A travers ce corpus et cette problématique de l'enfermement, nous nous sommes d'abord donné comme but de monter que les films marocains des années 1990 - 2000 constituent une unité et un corps homogène. En effet, à travers ses figures de proue, Saâd Chraïbi, Daoued Ouled Syad et Farida Benlyazid, la deuxième génération du cinéma marocain s'est employée à transgresser les barrières de toutes sortes, établies par la société qui clôture l'espace.

Saisi comme un signe au sein du système textuel, avec sa force signifiante et sa valeur, l'espace a nettement déterminé sa fonction de création d'un univers sémantique, dont les figures formelles et expressives structurent la clôture t l'enfermement .

Aussi, est-il légitime d'interroger la pratique cinématographique de cette nouvelle génération dans ce qu'elle considère comme étant son essence : son rapport au réel en termes de langage et d'engagement social.

Le grand mérite de certains cinéastes de la deuxième génération, notamment Sâad Chraïbi, Ouled Syad et Benlyazid, est d'avoir compris et fait comprendre au spectateur marocain que le cinéma est d'abord une question de regard. En affirmant que les films donnent à voir, ils ont souscrit évidemment à une évidence. Mais cette évidence n'en était pas une dans le contexte du cinéma marocain des années 1990, ni d'ailleurs dans celui de la cinématographie arabe en général. Aussi, cette évidence a-t-elle eu l'effet d'une mutation considérable. Cette évolution est décisive parce qu'elle a achevé l'affranchissement du cinéma marocain de la structure discursive pour le doter d'une structure optique adéquate.

Dans cet élan de modernité dont le réalisme s'est avéré être l'indispensable vecteur, nous avons vu la fraîcheur et la pertinence et des démarches et des choix esthétiques. Cette option d'un "gain de réalité » nous a paru être la principale requête faite par cette nouvelle vague. Dans leurs premières réalisations, les cinéastes ont entamé une rupture avec les modes de représentations conventionnels et notamment les stratégies narratives classiques. Ils ont délibérément renoncé au récit linéaire, à la tentation des événements spectaculaires, aux intrigues classiques plus ou moins ficelées, et aux héros à statut extraordinaire incarnés par des comédiens du théâtre, qui jusque là sévissaient, en dépit de quelques voix singulières, dans la pratique cinématographique nationale. Les cinéastes de la deuxième génération se sont rassemblés ainsi autour d'une même vision : " plus de réalité ». Cette vision s'est concrétisée dans les premiers films de Chraïbi, Ouled Syad et Benlyazid, mais aussi dans d'autres premiers films, à 
l'instar d'Abdelkader Lagtae (1991) et la plage des enfants perdus de Jillali Ferhati (1991).

Il apparaît donc que les films du corpus, et à travers eux l'ensemble de la pratique de la deuxième génération, sont principalement identifiables par une position commune qui s'insurge contre un cinéma qui résorbe le réel dans la représentation et dans la discursivité.

Aussi, la production cinématographique de la décennie, 1990 - 2000 est-elle riche de longs métrages de qualité, évidemment les films du corpus mais également d'autres réalisations à l'instar de Tresses de Jillali Ferhati, Mektoub de Nabyl Ayouch, la falaise de Fawzi Bensaidi, Le dernier spectacle de Nour-Eddine Lakhmari et Lalla Hobby de Mohamed Abderrahman Tazi. Seulement, l'effervescence de cette période autour de quelques films-phares ne peut dissimuler la précarité esthétique d'une large partie de la production. En effet, plus de la moitié des films ont été soit une déception artistique, soit un échec commercial, sinon un cumul des deux torts. Le cas des films de Hakim Noury nous semble à ce propos fort éloquent. En 1985, le cinéaste réalisateur de son premier long métrage, le facteur (1980), met en scène le marteau et l'enclume. Le récit du film est inspiré de la vie du personnage Tahar, âgé de 55 ans et l'archétype de fonctionnaire modèle qui menait une vie tranquille malgré les problèmes propres à sa modeste condition. Le film renoue alors avec une certaine tradition du cinéma marocain des années 70 qui œuvrait, avec beaucoup de maladresses, à glorifier la lutte du peuple de ce pays et dont le réalisateur Noury était le chef de file. Le Marteau et l'enclume illustre la parfaite continuité d'une thèse univoque et d'un langage formel à peine esquissé. Aussi, si le cas de Noury semble anecdotique, il n'empêche que la décennie 1990- 2000 a eu son lot considérable de films contestés et contestables à l'instar de la fête des autres, de Hassan Benjelloun (1990), La plage des enfants perdus de Jillali Ferhati (1991), Histoire d'une rose de Abdelmajid El Rchich (2000) et les Casablancais de Abdelkader Lagtae (1998).

Il n'est pas étonnant alors de voir que cette évolution qualitative n'a pas touché d'une manière organique la pratique cinématographique marocaine de la période en question. En effet, il n'existe pas d'écoles de formation spécialisées au Maroc, et tous les cinéastes marocains n'ont pas eu la chance de se former à l'étranger. Par contre, ce qui semble le plus caractéristique de la période, et le moins compréhensible, c'est la thématique consensuelle qui couvre les contenus de la quasi-totalité des films : celle d'une société marocaine traditionnelle, irrémédiablement coupée de la modernité. Une thématique qui perdurera au-delà des années 1990 - 2000.

En effet, la quasi majorité des films de ces deux dernières décennies a pour contexte sociopolitique un passé plus ou moins lointain, celui des premières années de l'indépendance, du protectorat français ou encore des 
époques bien plus reculées dans le temps. C'est le cas, tel que nous l'avons vu, de Ruses de femmes (1999) mais également de nombreux autres films, parmi lesquels le résistant Inconnu (1995) de Larbi Bennani, L'Ombre du pharaon de Souheil Benbarka , Les Cavaliers de la gloire (1993) du même réalisateur ( sorti sous un deuxième titre Les tambours de feu) et récemment Abdouchez les Almohades ( 2006) de Saïd Naciri.

Dans le cas des films du corpus, le souci accordé à la qualité esthétique, la primauté des thèmes soulevés ont fait que ces films ont réussi à répondre à ce double impératif de la rupture esthétique et thématique, revendiquée par l'ensemble des cinéastes de la nouvelle génération. Seulement, comment expliquer la persistance de ce regard passéiste et la récurrence harassante de l'image d'une société marocaine écrasée par les valeurs traditionalistes et rétrogrades ? Ce discours qui perdure tout au long de la décennie 1980 - 1990 reflète-t-il la société marocaine ? Est-ce le triste constat d'une démission collective du sens de l'histoire qui fait échouer la société marocaine dans un présent sans issue, celui d'une identité coincée entre une logique et une attitude mono-culturelle et passéiste ?

Depuis Vaincre pour vivre d'Ahmed Mesnaoui et Mohammed Tazi (1968) et jusqu'à w.w.w : What a wonderful world (2006), mille mois (2005) de Faouzi Bensaïdi et la couleur du sacrifice (2006) de Mourad Boncif, en passant jusqu'à en faire des clichés les images d'hommes et a fortiori de femmes assujettis, otages de structures traditionnelles et conservatrices. Même si on admet que pour la libération de l'individu, la cause individualiste ne serait- elle pas un simple paravent?

Certes, les cinéastes de la deuxième génération ont bien inauguré une remise en question de l'ordre moral établi. En posant le sujet, ils ont posé le Désir, l'enjeu étant principalement d'affranchir le corps du sujet de l'identification au père. L'acquis a été de revisiter l'image du père arabe, de la démystifier et parallèlement de remettre en question la capacité de la morale, par le biais de la famille, à canaliser les pulsions et les affects. Ainsi, les films échappent à l'impératif moral et à la résorption dans le symbolique pour peu que le sujet renoue avec le monde originel des pulsions, des émotions et des affects. Même si les cinéastes n'ont pas doté le sujet d'une capacité de transgression réelle, d'une affirmation de liberté, il n'en demeure pas moins vrai qu'ils ont le mérite d'avoir fait advenir à l'écran la structure renversée du regard spéculaire. A la faveur de ce nouveau rapport, les films ont pu donner à voir ce que refoulait jusque-là le système de représentation aussi bien cinématographique que télévisuel en vigueur depuis au moins trois décennies. Les cinéastes ont visé ainsi l'élargissement du champ d'élection des processus identificatoires que le cinéma investit. Le public marocain a entièrement adhéré. 


\section{References:}

1. Adieu Forain de Daoued AOULED SYAD,

2. CAILLOIS R., l'homme et le sacré, Paris, éd.Gallimard, collection Idées,1989.

3. Collectif, l'Enfermement, éd. Presses universitaires de Lille,1981.

4. DELEUZE. Gilles, Différence et répétition, Paris, PUF,1968.

5. DELEUZE. Gilles, l'image-mouvement, Paris, éd. de Minuit, 1983.

6. ESCOBAR MOLINA A., l'Enfermement : Espace-Temps Clôture, Paris, éd.Klincksieck, 1989.

7. Femmes et Femmes de Saâd CHRAÏBI

8. GOLDMAN A., Quelques problèmes de sociologie du cinéma (in sociologie et cinéma), Ed.les presses de l'Université de Montréal,1976.

9. Jawhara, fille de prison de Saâd CHRAÏBI,

10. PANKOW G., l'homme et son espace vécu, Paris, Éd. Aubin, 1986

11. .SORLIN Pierre, de l'Audiovisuel, Ed. Nathan Université, 1992.

12. Ruses de femmes de Farida BENLYAZID 